\title{
Empiema del Muñón Ureteral: Secretos Para un Buen Diagnóstico
}

\section{Empyema of the Ureteral Stump: Secrets for a Correct Diagnosis}

\author{
Federico Díaz Telli ${ }^{1}$ Eduardo Nicolás Mendes ${ }^{1} \quad$ Juan Francisco Trentacoste ${ }^{1}$ Jonathan Rosenvasser ${ }^{1}$ \\ Carlos Juan Padin ${ }^{1}$ Juan Pablo Perotti ${ }^{1}$ \\ ${ }^{1}$ Servicio de Diagnóstico por Imágenes Hospital Universitario Austral, \\ Presidente Derqui, Pilar, Buenos Aires, Argentina \\ Address for correspondence Federico Díaz Telli, MD, Servicio de \\ Diagnóstico por Imágenes, Hospital Universitario Austral, Presidente \\ Rev Argent Radiol 2019;83:71-76. \\ Derqui, Pilar, CP 1629, Buenos Aires, Argentina \\ (e-mail: federico@diaztelli.com.ar).
}

\section{Resumen \\ Palabras clave \\ - empiema \\ - nefrectomía \\ - infección \\ - dolor lumbar}

\section{Abstract}

El muñón ureteral es el segmento de uréter remanente posterior a una nefrectomía, que puede, ocasionalmente, dar origen a un cuadro sintomático infeccioso poco frecuente, conocido como empiema del muñón ureteral (EMU). El mismo suele atribuirse a otra patología por desconocimiento médico y no es reconocido hasta que el cuadro clínico avanza significativamente o se realiza la exploración quirúrgica. Los muñones ureterales que se encontraban sanos en la cirugía inicial no suelen desarrollar patología. Por el contrario, los uréteres obstruidos, crónicamente infectados o asociados a litiasis o reflujo distal, son los que se encuentran en riesgo de desarrollar complicaciones futuras.

En esta revisión, se repasa la literatura y se presentan casos de pacientes con antecedentes de nefrectomía que por diferentes causas transcurrieron con empiema en el muñón ureteral, con el fin de analizar las posibles causas y factores predisponentes de la patología, describir los hallazgos radiológicos en los diferentes métodos diagnósticos y poder reconocer las posibles complicaciones para su correcto manejo terapéutico.

Las infecciones urinarias a repetición son útiles para sospechar la presencia de EMU. Ellas se deben al reflujo urinario o disfunción en el vaciamiento del uréter remanente, con estasis e infección del mismo. Por lo tanto, en pacientes con antecedentes de nefrectomía que presentan dolor abdominal difuso, fiebre y antecedentes de infecciones urinarias a repetición, es necesario sospechar empiema del muñón ureteral para poder realizar un correcto análisis imagenológico y posterior tratamiento.

The ureteral stump is the segment of the ureter that remains after a nephrectomy, and it can occasionally give rise to a rare symptomatic infectious disorder known as an empyema of the ureteral stump. The syndrome is usually attributed to another disease due to the radiologist's or ED physician's unawareness, and diagnosis is delayed until there is significant clinical progression, complimentary images or exploratory surgery is performed.

Ureteral stumps that were healthy at the initial surgery, usually do not pose further problems. On the other hand, ureters that are obstructed, chronically infected or received October 4, 2018 accepted May 6, 2019
DOI https://doi.org/ $10.1055 / \mathrm{s}-0039-1692152$. ISSN 1852-9992.
Copyright @ 2019 , Sociedad Argentina de Radiología. Publicado por Thieme Revinter Publicações Ltda., Rio de Janeiro, Brazil. Todos los derechos reservados.
License terms

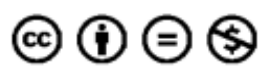




\section{Keywords}

- empyema

- nephrectomy

- infection

- low back pain associated with nephrolithiasis or distal reflux, are at risk for developing future complications.

This review goes over the available literature on the subject and presents cases of patients who underwent a nephrectomy and, for different reasons, developed an empyema of the ureteral stump in order analyze the possible causes and predisposing factors, describing the main radiologic findings in each of the different imaging modalities, and recognize the possible complications and their according therapeutic management.

Repeated urinary tract infections are useful for suspecting the presence of an empyema, and are due to vesicoureteral reflux or ureteral dysfunction, with consequent urinary stasis and infection. Thus, patients with a history of nephrectomy presenting with diffuse abdominal pain, fever and repeat urinary infections, should raise the suspicion of empyema of the ureteral stump, leading to a correct imaging analysis and posterior treatment.

\section{Introducción}

El clásico manejo terapéutico de un riñón no funcionante o pobremente funcionante es la nefrectomía total con ureterectomía proximal, persistiendo en la mayoría de los casos un remanente ureteral, que involucra el tercio medio y distal del uréter.

El empiema del muñón ureteral (EMU), es una patología rara y poco conocida tanto por el médico emergentólogo como por el radiólogo, que consiste en la infección del remanente ureteral, con una incidencia reportada de entre el $0,8 \%$ y el $1 \%{ }^{1,2}$

En ocasiones, el empiema ureteral puede ser el origen de un proceso inflamatorio e infeccioso inespecífico con sintomatología variada y de difícil diagnóstico, ya que en la mayoría de esos casos, dicha sintomatología no se asocia a causas urológicas, dificultando el diagnóstico o sospecha diagnóstica y retrasando el tratamiento adecuado. ${ }^{3}$ Generalmente, el muñón ureteral no suele presentar patología si al momento de la cirugía no se encontraba asociado a alteraciones, mientras que los uréteres crónicamente infectados, obstruidos, asociados a litiasis o a reflujo distal son los que pueden producir sintomatología. El intervalo de tiempo entre la intervención quirúrgica y la aparición de los síntomas puede variar entre meses y varios años.

Tiempo atrás, la enfermedad ureteral era estudiada con radiología convencional por ureteropielografía ascendente, mientras que en la actualidad la ecografía, tomografía computada (TC) o resonancia magnética (RM) la han reemplazado y a pesar de no mostrar el reflujo ureteral de forma dinámica, evalúan de mejor manera sus complicaciones. Hoy en día, el diagnóstico tiende a ser más precoz, aun cuando la enfermedad no es inicialmente sospechada, debido al amplio uso de la TC para patología

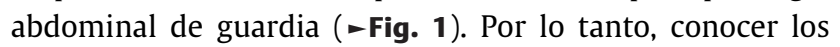
hallazgos imagenológicos y las opciones terapéuticas contribuye al manejo de una enfermedad poco conocida.
Presentamos tres casos con sintomatología inflamatoria lumbar asociados a un cuadro infeccioso de origen desconocido y todos ellos nefrectomizados por distintas causas (hipoplasia renal con riñón no funcionante, por dilatación crónica del uréter secundaria a un implante pelviano y por litiasis e infecciones urinarias a repetición).

\section{Hallazgos Imagenológicos}

En la actualidad, la ureteropielografía ascendente ( - Fig. 4) se encuentra mayormente fuera de uso para el diagnóstico de esa patología, aunque podría llegar a ser un método diagnóstico para certificar el cuadro patológico, demostrando en la mayoría de los casos el uréter remanente dilatado, de morfología lobulada y en algunos casos evidenciar reflujo vesicoureteral como punto de partida de la enfermedad.

La ecografía es ampliamente utilizada como estudio diagnóstico inicial debido a su gran utilidad y disponibilidad en el servicio de emergencias. Puede orientar el diagnóstico en el ojo de un radiólogo entrenado y que sospeche del cuadro sintomático a partir de los antecedentes de enfermedad. Permite visualizar el trayecto del uréter inflamado, aunque no siempre se visualice en su totalidad debido a la posición profunda que el mismo adopta en su ubicación retroperitoneal. Además, es útil para descartar otras posibles patologías que generen dolor lumbar o pelviano asociados a un cuadro infeccioso ya sea de causa ginecológica (salpingitis, EPI) o gastrointestinal (colitis, apendicitis, diverticulitis). ${ }^{4-8}$

La RM (-Fig. 5) puede mostrar una mejor resolución espacial del cuadro inflamatorio en comparación con la ecografía, evidenciando un aumento en el calibre del uréter remanente, así como también el engrosamiento de sus paredes, la inflamación regional asociada con sus posibles causas y complicaciones. Raramente se utiliza la RM para certificar el cuadro diagnóstico, ya que no es tan ampliamente utilizada en urgencias abdominales y su detección por este método suele ser un hallazgo incidental. ${ }^{4-8}$ 


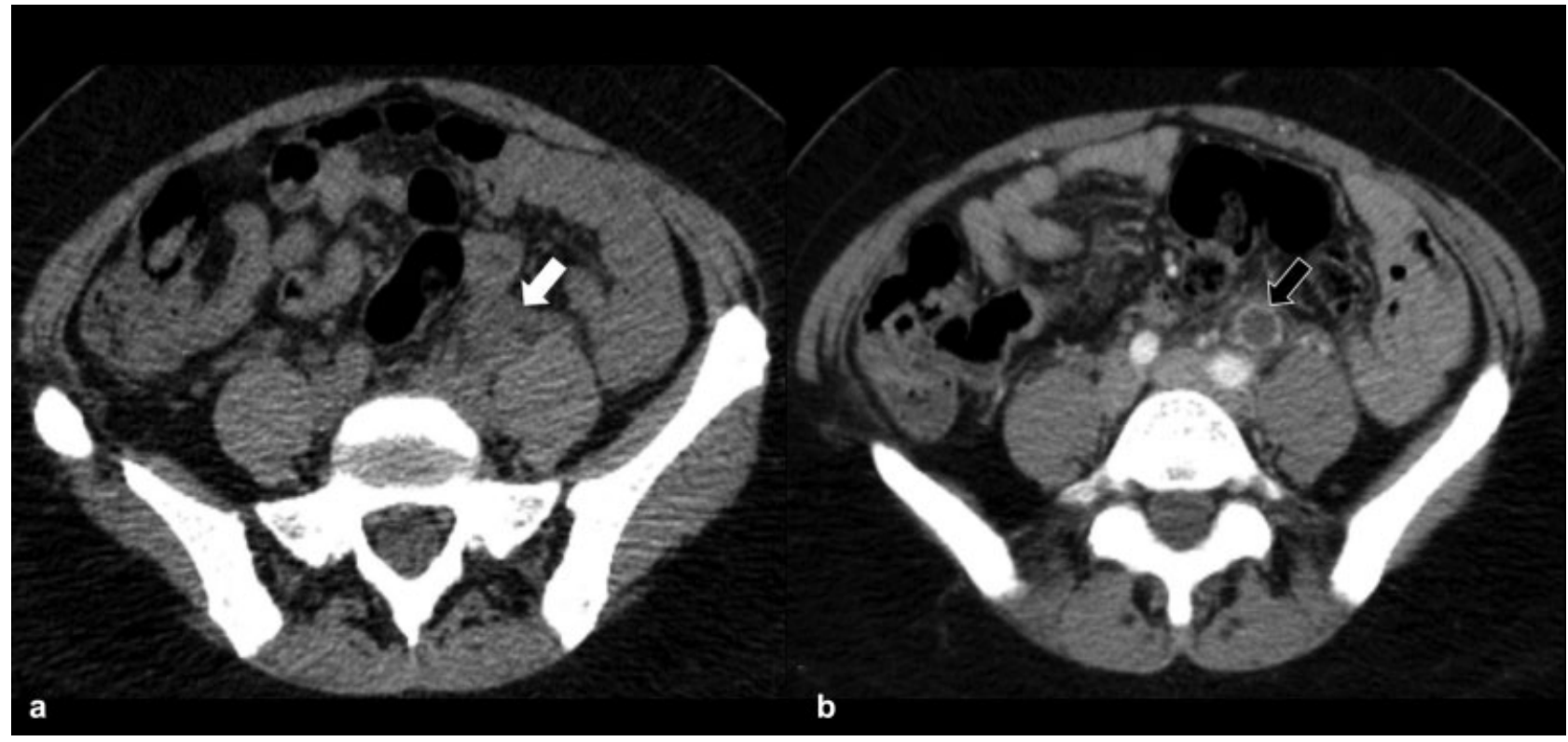

Fig. 1 Paciente con nefrectomía izquierda por infección del tracto urinario (ITU) y litiasis a repetición. TC de abdomen sin contraste en corte axial (a) donde se reconoce proceso inflamatorio retroperitoneal izquierdo, de difícil caracterización (flecha blanca). TC de abdomen con contraste endovenoso en corte axial (b) que muestra una estructura tubular dilatada retroperitoneal izquierda, con refuerzo de sus paredes y cambios inflamatorios regionales, compatible con empiema del muñón ureteral (flecha negra).

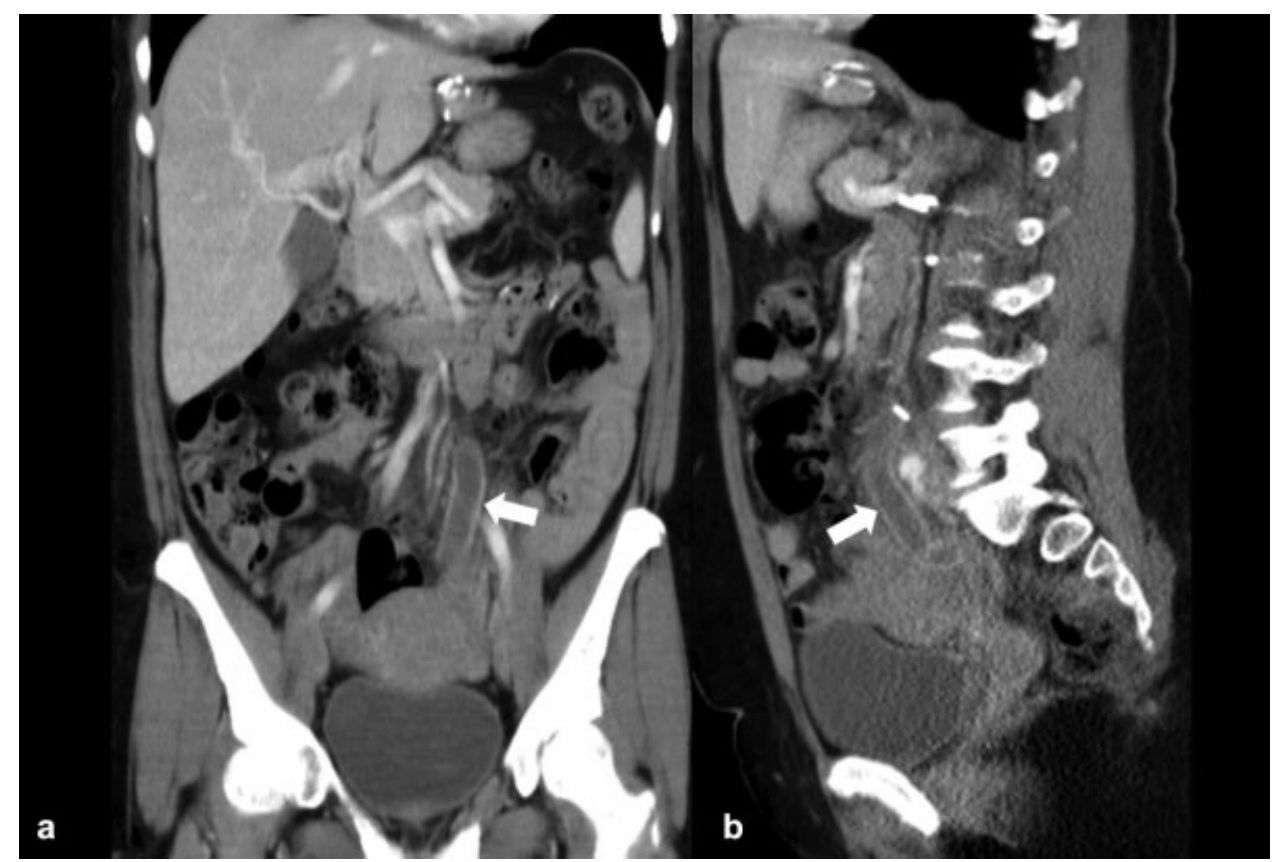

Fig. 2 Reconstrucciones tomográficas en (a) coronal y (b) sagital del caso descripto en la — Figura 1. Se reconoce la dilatación del uréter remanente con refuerzo de sus paredes a lo largo de todo su trayecto (flechas blancas).

La TC con contraste endovenoso es por amplio margen la mejor técnica para el diagnóstico del EMU. Se trata de un estudio rápido, con alta sensibilidad y especificidad, que nos permite ( - Fig. 2) no solo demostrar la existencia de un EMU visible como una estructura tubular dilatada en el trayecto del uréter remanente, con refuerzo y engrosamiento de su pared, sino que también nos ayuda a evaluar las posibles causas del mismo (litiasis, compresión extrínseca de la pared del muñón o tumores) y complicaciones como abscesos asociados en peritoneo o en músculo psoas ( - Fig. 3$)^{4-8}$

\section{Discusión}

Lo más importante del cuadro diagnóstico es reconocer la probable existencia de la patología en pacientes con clínica inflamatoria lumbar, asociados a sintomatología infecciosa y 


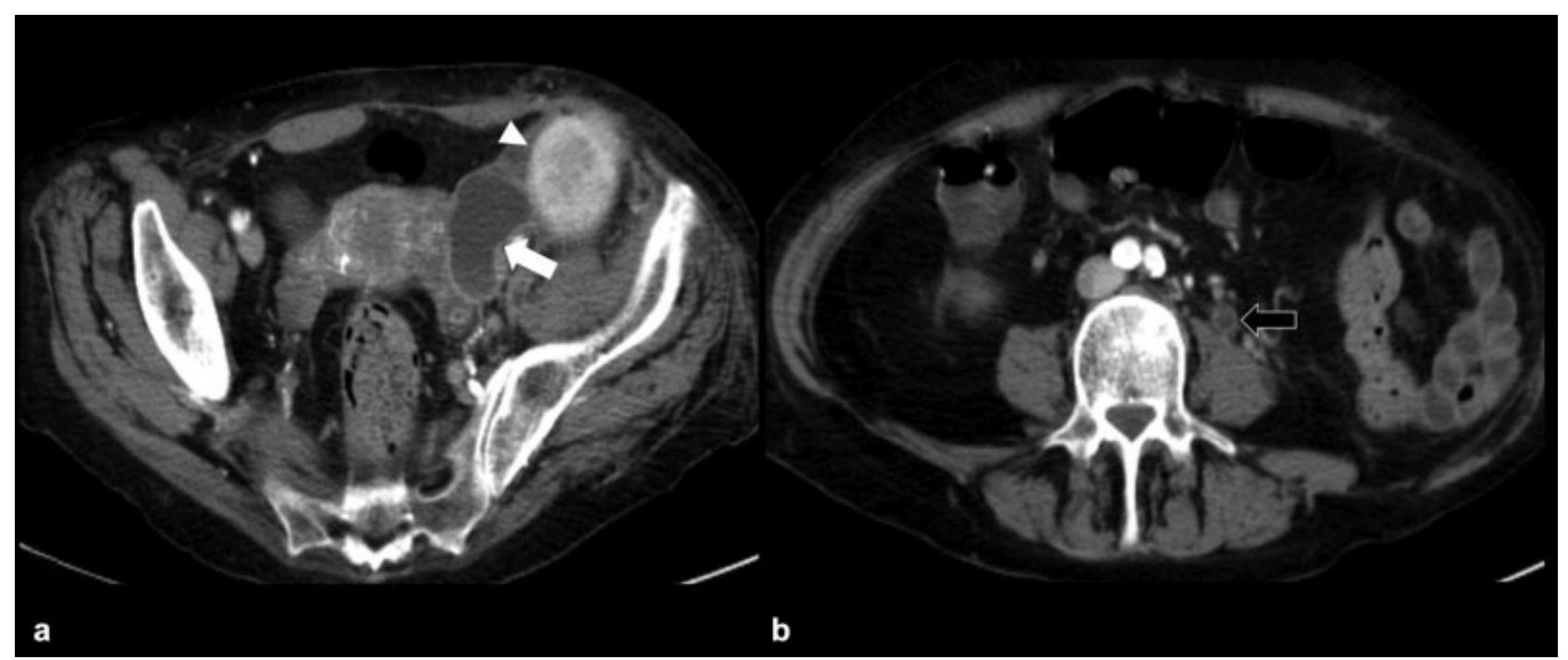

Fig. 3 Paciente trasplantado renal. TC de pelvis con contraste endovenoso (a) en donde se reconoce colección con refuerzo de sus paredes (flecha blanca) adyacente al riñón trasplantado (cabeza de flecha). TC de abdomen del mismo paciente (b), evidenciándose dilatación e inflamación del muñón ureteral izquierdo remanente (flecha negra).

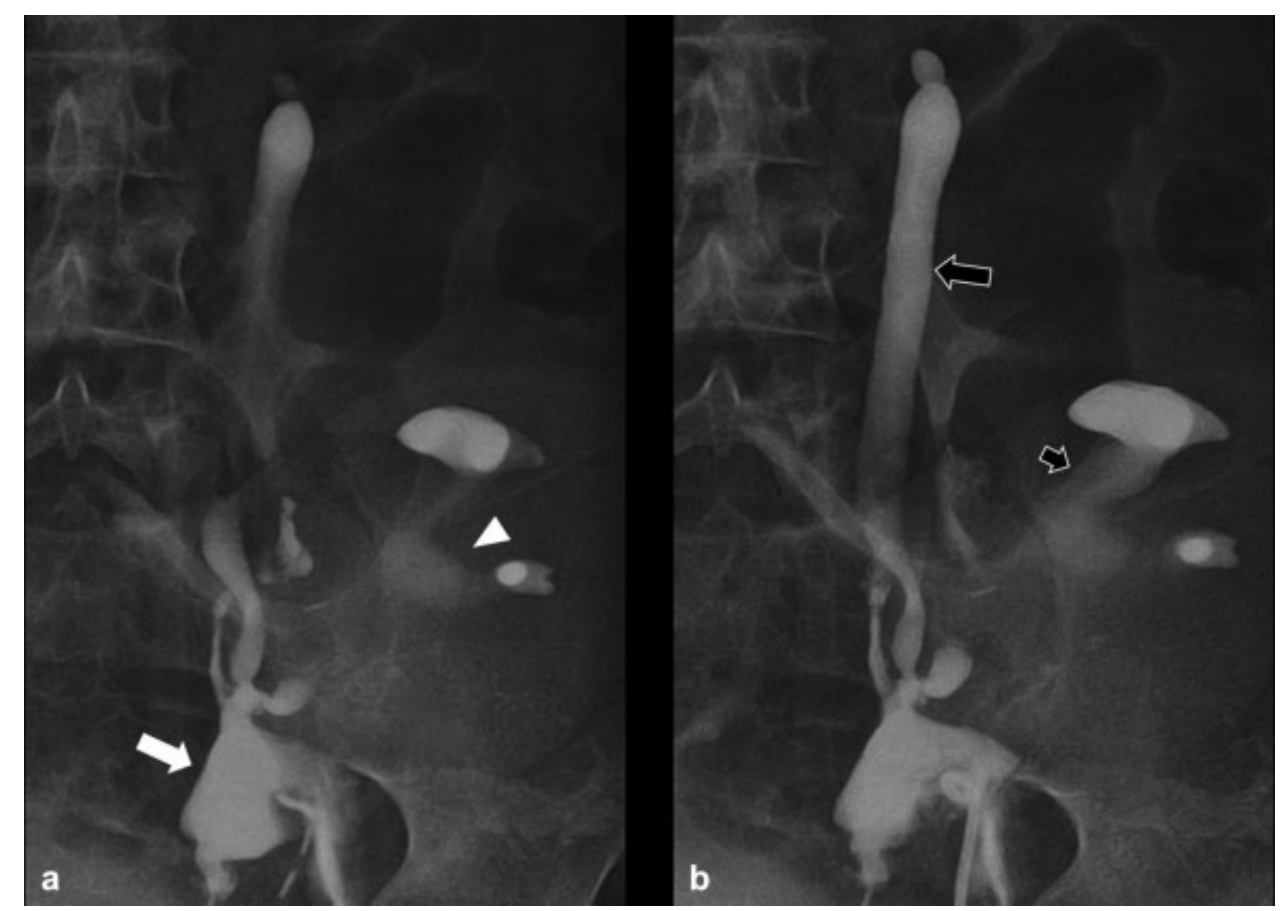

Fig. 4 Fistulografía en paciente con riñón trasplantado en fosa ilíaca izquierda. (a) Colección periureteral (flecha blanca) por delante del riñón trasplantado (cabeza de flecha). (b) Dilatación del muñón ureteral en todo su trayecto (flecha negra) y dilatación del sistema uroexcretor del riñón trasplantado (flecha negra corta).

con antecedentes de nefrectomía. Si bien en la mayor parte de la bibliografía se describe un intervalo de tiempo lejano entre la nefrectomía y el cuadro inflamatorio, en nuestra institución se reconocieron dos casos de empiemas ureterales previos a los tres meses postquirúrgicos. ${ }^{9}$

$\mathrm{Si}$ bien todos esos pacientes fueron estudiados inicialmente por ecografía con hallazgos inespecíficos, la revisión bibliográfica revela que, en todos los casos reportados, la TC con contraste endovenoso es el método de referencia para el diagnóstico del EMU. Se comprobó que, al ojo de un radiólogo con conocimiento claro de la patología, el diagnóstico por TC se realiza rápidamente. No solo se certifica el cuadro inflamatorio, sino que se reconocen las posibles causas, enfermedades asociadas o complicaciones del empiema, en algunos casos reconociendo abscesos en las estructuras retroperitoneales adyacentes.

Es importante tener en cuenta que la sensibilidad y especificidad de la TC aumenta en un tomógrafo multislice en relación a un helicoidal, debido al menor espesor de corte 


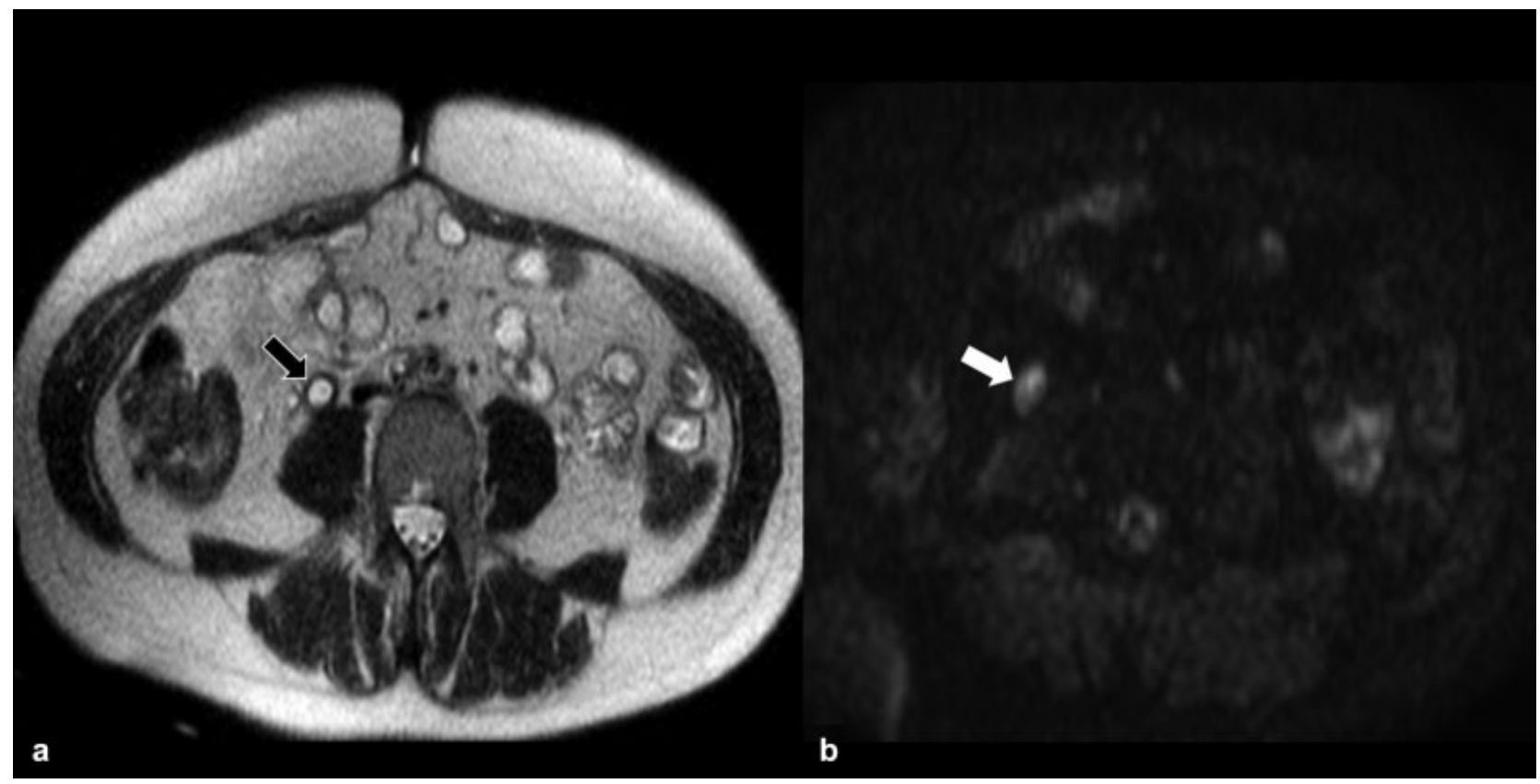

Fig. 5 Paciente con nefrectomía derecha por implante pelviano secundario a un carcinoma de cérvix. (a) RM de abdomen en secuencia ponderada en T2, donde se reconoce uréter derecho dilatado, de paredes engrosadas con contenido líquido en su interior (flecha negra). En la secuencia en difusión del mismo paciente (b), se identifica inflamación del uréter remanente en secuencias de difusión (flecha blanca).

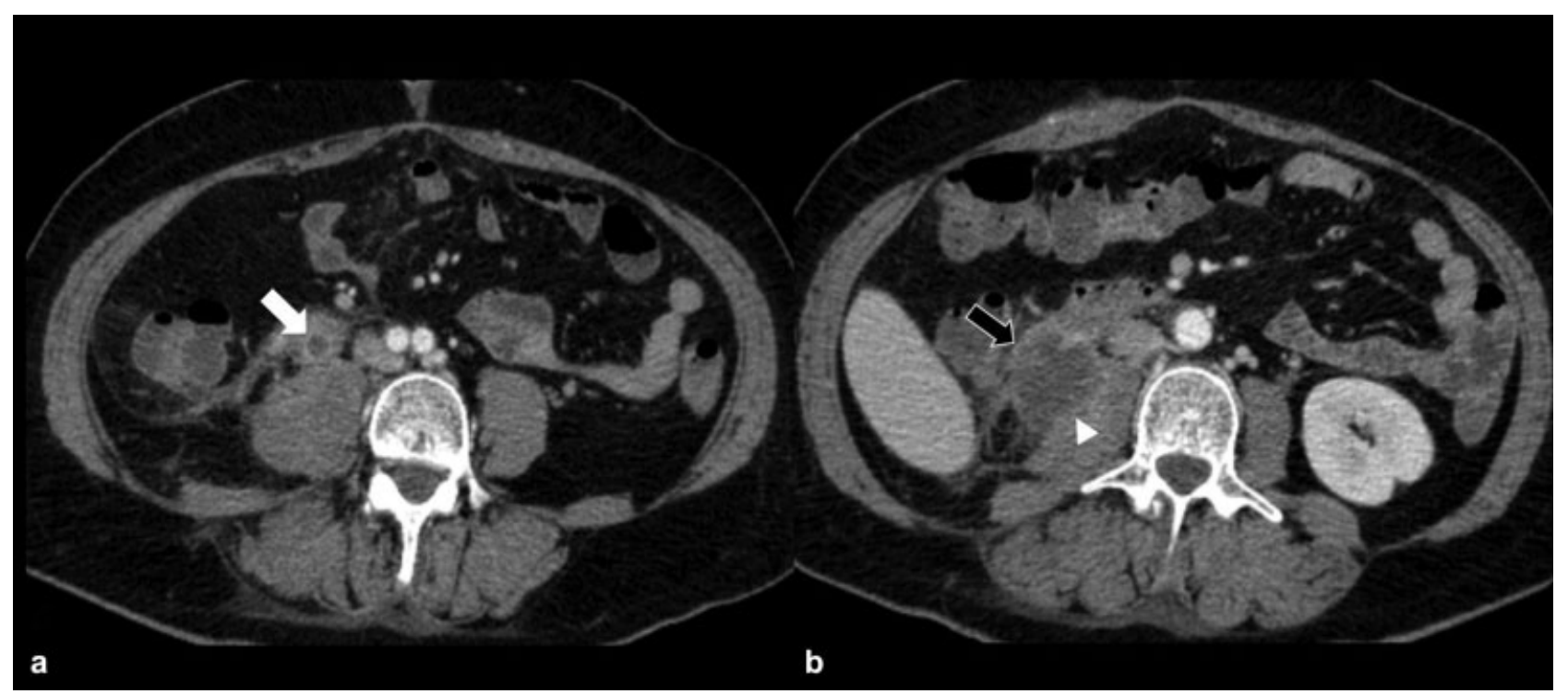

Fig. 6 TC de abdomen con contrate endovenoso donde se observa en (a) uréter remanente derecho dilatado, con cambios inflamatorios adyacentes (flecha blanca). (b) Colección organizada originada en el extremo caudal del uréter remanente (flecha negra), con afectación del músculo psoas homolateral (cabeza de flecha).

y a las reconstrucciones multiplanares. Los hallazgos tomográficos característicos muestran una estructura tubular retroperitoneal dilatada, con refuerzo de sus paredes, en topografía del uréter remanente, con contenido líquido en su interior, en pacientes con el antecedente de nefrectomía homolateral. Una vez realizado el diagnóstico, es necesario evaluar las posibles causas de enfermedad, descartando litiasis, tumor o patología inflamatoria genitourinaria. Desde el punto de vista terapéutico, en la mayoría de los casos se realizará tratamiento antibiótico empírico con posible resolución quirúrgica en un segundo tiempo según su evolución. ${ }^{10-12}$ En algunos casos, se realizará tratamiento intervencionista con un drenaje percutáneo en pacientes con colecciones extraureterales asociadas (-Fig. 6).

\section{Conclusión}

Por todo lo expuesto anteriormente, se considera que el diagnóstico de un EMU requiere un alto grado de sospecha clínica en pacientes con el antecedente de nefrectomía asociado a dolor lumbar y a un cuadro infeccioso de 
etiología incierta, para así poder tener un diagnóstico precoz. $^{13}$ La TC es el método de elección para la evaluación de esos pacientes, ya que no solo permite identificar con claridad el proceso inflamatorio y sus complicaciones regionales, sino que también ayuda a evaluar posibles causas predisponentes a la enfermedad y probables diagnósticos diferenciales a la misma. En nuestra experiencia, es importante para el radiólogo conocer la patología para así poder realizar un adecuado manejo terapéutico de los pacientes en la emergencia.

\section{Responsabilidades Éticas}

Protección de personas y animales. Los autores declaran que para esta investigación no se han realizado experimentos en seres humanos ni en animales.

Confidencialidad de los datos. Los autores declaran que han seguido los protocolos de su centro de trabajo sobre la publicación de datos de pacientes.

Derecho a la privacidad y consentimiento informado. Los autores declaran que en este artículo no aparecen datos de pacientes.

Conflicto de Intereses

Los autores declaran no tener ningún conflicto de intereses.

\section{Bibliografía}

1 Krarup T, Wolf H. Refluxing ureteral stump. Scand J Urol Nephrol 1978;12(02):181-183
2 Labanaris AP, Zugor V, Smiszek R, Nützel R, Kühn R. Empyema of the ureteral stump. An unusual complication following nephrectomy. ScientificWorldJournal 2010;10:380-383

3 Androulakakis PA, Stephanidis A, Antoniou A, Christophoridis C. Outcome of the distal ureteric stump after (hemi)nephrectomy and subtotal ureterectomy for reflux or obstruction. BJU Int 2001;88 (06):586-589

4 Labairu-Huerta L, Burguete-Moriones A, Zabalza-Unzué J, GrasaLanau V. Empiema del muñón ureteral. A propósito de un caso y revisión de la literatura. Actas Urol Esp 2010;34(10):909-911

5 Pollack HM, Banner MP, Popky GL. Radiologic evaluation of the ureteral stump. Radiology 1982;144(02):225-230

6 Persad R, Kamineni S, Mouriquand PD. Recurrent symptoms of urinary tract infection in eight patients with refluxing ureteric stumps. Br J Urol 1994;74(06):720-722

7 Prajsner A, Szewczyk W, Gawron R. Empyema of retained ureteral stump with gross stone 20 years after nephrectomy. Urology 2009;73(05):993-994

8 Ikeda D, Matsutani R, Fukuda M, Fuse H, Hirano S. Transurethral fulguration for empyema of ectopic ureteral stump. Int J Urol 2003;10(12):664-666

9 Bulotta AL, Ferrara F, Sica M, Di Maggio G, Messina M. Distal Ureteral Stump. JSAS 2011;3:58-60. Doi: 10.4081/jsas.2011.574

10 Ehrlich RM, Koyle MA, Shanberg AM. A technique for ureteral stump ablation. J Urol 1988;140(5 Pt 2):1240-1241

11 Suzuki T, Tsuchiya N, Otomo R, et al. Primary tumor of the ureteral stump following a nephrectomy for renal cell carcinoma. Int J Urol 1999;6(01):41-43

12 Cain MP, Pope JC, Casale AJ, Adams MC, Keating MA, Rink RC. Natural history of refluxing distal ureteral stumps after nephrectomy and partial ureterectomy for vesicoureteral reflux. J Urol 1998;160(3 Pt 2):1026-1027

13 Barón Ródiz PA, Pérez De Los Ríos A, Pérez Dávila M, Concejo Iglesias P, Poyo Calvo JC. No nos olvidemos del muñón ureteral. SERAU 2017;241 ID: C2017-241 